\title{
EDITORIAL
}

\section{CHILDHOOD BLINDNESS IN INDIA}

The article by Rahi and colleagues in this issue seeks to assess the causes of child blindness in India from a survey of children in blind schools. Child blindness, especially if it is preventable, is a particularly emotive subject and there are several important issues raised by this article.

From the results of this survey, vitamin A deficiency causing xerophthalmia is still the most important single cause of childhood blindness in India and there are good reasons to think that this survey may underestimate the true significance of vitamin A deficiency in the community. Firstly, the authors only examined children in blind schools and they estimate that there are approximately 200000 blind children in India of whom only 20000 are receiving appropriate education. It is likely that the minority of children who receive appropriate education in blind schools are more privileged than the rest and so would be less likely to have vitamin A deficiency. Secondly, the survey also excluded pre-school age children, which is the age group most susceptible to vitamin A deficiency. Thirdly, in the last decade epidemiologists have realised that corneal ulceration and scarring represents only the visible tip of the iceberg of vitamin A deficiency. These children are more susceptible to a wide range of infections and in particular a greatly increased risk of death. A recent WHO report has estimated that world-wide 5-10 million children a year develop xeropththalmia of which a very significant proportion go blind. ${ }^{1}$ If the situation as regards xerophthalmia is bad in India it is worse in many parts of Africa, where up to three-quarters of child blindness may be related to vitamin A deficiency and measles.

The link between vitamin A deficiency and child blindness has been known for many years and also that vitamin A deficiency causes a greatly increased susceptibility to infections and in particular measles. Both vitamin A deficiency and measles can be prevented at very little financial cost to the community and yet they still remain the most important cause of world-wide childhood blindness. There is a positive side to the statistics about vitamin A deficiency in this article. In one Indian state, Kerala, vitamin A deficiency was the cause of only $7 \%$ of the blindness as opposed to $26 \%$ in Madya Pradesh. So it would seem that prevention of xerophthalmia programmes can work effectively in some parts of India.

The authors also commented on the problem of consanguinity as a cause of blindness through the homozygous expression of recessive genes. They estimated this to be the cause of $23 \%$ of child blindness. Marrying close relatives is common in certain communities, and the eye as both a complex and accessible organ is both likely to be affected by recessive genes and to produce changes that can be readily seen. This is obviously an area which needs further study, but even more importantly local ophthalmologists need encouragement to speak out against such marriage arrangements and warn of their dangers. Marital consanguinity has many complex cultural and social causes. In general those who encourage it think that it prevents 'bad blood getting into the family', little realising that it does just the opposite.

Congenital cataract was the cause of $12 \%$ of the blindness, with problems of untreated cataract, amblyopia from delayed surgery and post-operative surgical complications. It is not possible to know what proportion this represented of all the children with congenital cataract, as those who were successfully treated obviously would not be educated in blind schools. It is, however, a significant proportion of blind school children. India with its vast problem of adult cataract has great expertise in cataract surgery, but the much less frequent congenital cataract in children requires different and more exacting treatment which may only be available in special units. Early surgery is essential as is optical correction of the aphakia. It would appear again that much of the congenital cataract is related either to consanguinity or to rubella infection in pregnancy and so could be prevented.

The authors estimate that the prevalence of child blindness in India is 5 times that in the developed world. From where will the impetus come to do something to reduce this? It may well be that the ultimate responsibility rests with government health authorities, but some must also rest with individual 
ophthalmologists. It is very easy for those of us working in economically privileged countries to be critical of ophthalmologists and government health departments in developing countries. The truth is that most ophthalmologists cannot survive on a government salary working in poorly equipped and badly staffed hospitals and clinics and are obliged to turn to private practice in the major cities for their livelihood.

It would seem essential for every ophthalmologist working in a developing country to be involved in blindness prevention, community outreach and health education - activities which have a very different emphasis to private medical practice. Unfortunately, most of us have very little training in these skills and there is an urgent need for reappraisal of postgraduate training both in developing countries and for those ophthalmologists from there who come to Western countries for postgraduate training before returning home. There is also an obvious need for both training and supporting paramedical workers in eye care in countries where there is often less than one ophthalmologist for a million people.

Finally, does this article have any message for the majority of this journal's readers who live and work in the developed world where children do not go blind from preventable disease? Vitamin A deficiency is so rare that individual cases get written up as case reports, ${ }^{2}$ parental consanguinity exists only in a few very isolated and immigrant communities, and prompt and effective specialist treatment is available for congenital cataracts. There is indeed no direct message, but there is perhaps an indirect one. As the costs of health care continue to rise and the demand for it also increases, especially in ophthalmology with its emphasis on the elderly, so in a much smaller way the Western world faces the problem of the developing world in that the demand for health care exceeds supply. Epidemiologists have shown us that especially in glaucoma and diabetic retinopathy, there is still a great deal of unrecognised and untreated but preventable loss of vision 'out there' in the community.

Faced with our own problem of preventable disease, of not being able to meet all the health needs of the community, and of increased waiting times for both outpatient clinics and surgery, it is not enough just to weep 'crocodile tears' or to expect 'the government to do something about it'. We must all be prepared to respond as imaginatively and creatively as we can to the needs of the community, to review our protocols for treatment, to maximise our utilisation of hospital resources, to consider 'shared care' schemes, technical and paramedical assistance, or anything that will make finite resources work as effectively as possible.

JOHN SANDFORD-SMITH

\section{References}

1. Sommer A. Vitamin A deficiency and its consequences. Geneva: WHO, 1995.

2. Watson MJ, et al. Vitamin A deficiency in xerophthalmia in the United Kingdom. BMJ 1995;310:1050. 